\title{
Tagungen
}

\author{
Milan Bulaty, Michael Müller-Preußker, Peter Schirmbacher
}

\section{Informations- und Kommunikationszentrum in Adlershof}

\section{Planungen und Thesen}

Die Humboldt-Universität zu Berlin plant, mit den mathematisch - naturwissenschaftlichen Instituten in die sich entwickelnde Wirtschafts- und Wissenschaftsstadt Berlin-Adlershof (WISTA) umzuziehen. Die Universität hat deshalb eine Reihe von Workshops ins Leben gerufen, bei denen sie die erarbeiteten Konzepte einschlägigen Fachleuten zur Diskussion stellt, um Anregungen zu Veränderungen oder Bestätigung von eingeschlagenen Wegen zu erhalten.

So sind auch die hier vorgelegten Thesen zum Workshop „Die Bibliothek der Zukunft, Planungen zu einem Informations- und Kommunikationszentrum in Adlershof" entstanden. Dieser Workshop fand am 11. Oktober 1995 in der Humboldt-Universität statt; einzelne Vorträge wurden im WWW-Server der Universitätsbibliothek veröffentlicht (http:/www.unibib.hu-berlin.de/welcome.html). Der Beitrag von Elmar Mittler mit dem gleichnamigen Titel wird ebenfalls in diesem Heft veröffentlicht.

Die hier vorgelegten Thesen berücksichtigen vor allem, daß die kommende Informationsversorgung der Humboldt-Universität sowie der außeruniversitären Forschungseinrichtungen und Wirtschaftsunternehmen gemeinsam nur durch eine enge Zusammenarbeit der Bibliothek und einer Zentraleinrichtung für Informationsverarbeitung und Kommunikation, dem ehemaligen Rechenzentrum, zu realisieren ist.

\section{Thesen zum Workshop}

1. Die naturwissenschaftlichen Institute der HumboldtUniversität werden voraussichtlich bis zum Jahre 2003 aus der Berliner Mitte an den Wissenschafts- und Wirtschaftsstandort Berlin-Adlershof ziehen. An diesem Standort werden dann eine Vielzahl außeruniversitärer Forschungsinstitute und vielfältige innovative Wirtschaftsunternehmen gemeinsam mit universitärer Forschung und Lehre konzentriert sein. Die intensive Kooperation der verschiedenartigen Einrichtungen miteinander und mit auswärtigen Partnern läßt erhebliche Synergie-Effekte in Forschung, Entwicklung und Produktion erwarten.

2. Die wichtigste Infrastruktur-Einrichtung für den Standort wird das gemeinsam mit der WISTA Management $\mathrm{GmbH}$ zu errichtende Informations- und Kommunikationszentrum Adlershof (IKA) sein. Es soll neben der Naturwissenschaftlichen Zentralbibliothek und der Zen- traleinrichtung für Informationsverarbeitung und Kommunikation (dem bisherigen Rechenzentrum) als wichtigste universitäre Elemente kommerzielle Informationsdienste, wissenschaftliche Verlage und die notwendige Infrastruktur eines solchen Komplexes (Telekonferenzund Besprechungsräume, Cafeteria u.a.) beherbergen. 3. Die Planungen zum IKA müssen die Möglichkeiten und Angebote modernster Informations- und Kommunikationstechnik genauso berücksichtigen wie die sich daraus ergebenden Anforderungsprofile der vielfältigen Nutzer. Das Zentrum muß in Aufgaben und Struktur den grundlegenden Entwicklungen auf den Gebieten der elektronischen Datenhaltung, des Datenverkehrs in Netzen, des elektronischen Publizierens Rechnung tragen. Es muß so konzipiert werden, daß es seinerseits als Informationsanbieter und -vermittler eine aktive Rolle am Wissenschafts- und Wirtschaftsstandort und weit darüber hinaus, d.h. weltweit, spielen kann.

4. Eine moderne Bibliothek wird ohne enge Kooperation mit Rechenzentrums- und Medieneinrichtungen nicht lebensfähig sein. Diesem Grundsatz müssen sowohl die inhaltliche als auch die Bauplanung für zukünftige wissenschaftliche Bibliothekseinrichtungen die entsprechende Beachtung schenken.

5. Die traditionellen bibliothekarischen Leistungen (Informationen auswählen, zur Verfügung stellen, erschließen, archivieren, Auskünfte erteilen und beraten) werden sich grundsätzlich nicht verändern. Sie werden aber in erster Linie auf elektronischen Medien basieren. Das betrifft zunächst den Nachweis, die Bestandshaltung und Pflege sowie die systematische Erschließung bereits vorhandener Buch- und Zeitschriftenbestände. Das gilt ferner für die Auswahl der Beschaffung und Einarbeitung neuer Informationsträger wie auch für die Kommunikation mit anderen Bibliothekseinrichtungen, Verlagen und Informationsanbietern.

6. Die Bibliothek wird sich auch in Zukunft mit dem Buch bzw. der gedruckten Zeitschrift beschäftigen müssen. Das gilt zunächst für die in der Vergangenheit angelegten und in naher Zukunft noch anzulegenden Bestände. Es bleibt offen, ob sich das systematische elektronische Scannen alten Schriftguts durchsetzen wird. Die Fernleihe einzelner nachgefragter Schriften kann allerdings auf diese Weise rationeller und ohne großen Zeitverzug für den Nutzer realisiert, und vor allem die Literatur kann direkt am Arbeitsplatz des Wissenschaftlers verfügbar 
gemacht werden. Große Wartezeiten auf Grund ausgeliehener Literatur gehören so der Vergangenheit an.

7. Gedruckte Literatur wird wohl noch lange ein Kulturgut bleiben, von dem sich die Leser nur sehr zögernd verabschieden werden, zumal die Lesbarkeit und die ständige Verfügbarkeit des bedruckten Papiers so schnell nicht übertroffen sein dürften. Daher wird die Bibliothek auch eine Schnittstelle darstellen müssen zwischen elektronischen und traditionellen Informationsträgern. Sie braucht leistungsfähige Druck- und Buchbindevorrichtungen, die der individuelle Nutzer aus Kosten- und Platzgründen nicht besitzen kann.

8. Neben die oben erwähnten traditionellen Aufgaben der Bibliothek treten neue. Die Möglichkeiten elektronischer Medien verändern bereits heute grundlegend die Kommunikation innerhalb einzelner Wissenschaftsdisziplinen. Das in der Hochenergiephysik vor mehr als 30 Jahren eingeführte und erprobte Preprintwesen wurde von anderen Disziplinen übernommen und hat nunmehr Einzug in die Computernetze gehalten. Damit verändert sich auch die verlegerische Tätigkeit grundlegend. Die Datenhaltung kann dezentral erfolgen, Nutzer können über Datennetze unmittelbar an die Daten bzw. Publikationen gelangen. Es wird möglich, die Informationen völlig neu zu strukturieren. Die Bibliothek kann damit auch zum direkten Konkurrenten in der verlegerischen Tätigkeit werden. Im Verein mit dem Rechenzentrum kann sie den Vertretern von Forschungsschwerpunkten im eigenen Hause die Betreuung von weltoffenen Datenbanken und Publikationssystemen auf eigenen Servern kostengünstig anbieten.

9. Die Bibliothek als allgemeines Informations-, Textund Multimediazentrum wird in Kooperation mit dem Rechenzentrum den Umgang mit neuen Medien zu propagieren und die Nutzer entsprechend zu schulen haben. Sie gibt Unterstützung beim Zugang zu weltweit verteilten Informationsressourcen und Hilfestellung bei der Recherche in verschiedenartigen, über die Datennetze verfügbaren Informationsbeständen.

10. Die Wissenschaft kann und wird nicht darauf verzichten, gesicherte Erkenntnisse und für die weitere Entwicklung als wertvoll anzusehende Forschungsleistungen von fehlerhaften und uninteressanten Aussagen zu trennen. Diese Auswahl mit Hilfe von Fachexperten, den Referees, zu treffen, gehört zu den wichtigen Aufgaben des Verlagswesens. Wenn auch Bibliotheken als Informationsanbieter im Auftrage der Institute verlegerische Aufgaben übernehmen, wird es möglicherweise zu neuen Verflechtungen von Wissenschaftseinrichtungen und ihren Bibliotheken mit Verlagsunternehmen kommen. Dieses ist ein interessanter Aspekt für die Entstehung von Informations- und Kommunikationszentren, wie für das in Adlershof geplante.
11. Das Datenkommunikationsnetz des naturwissenschaftlichen Campus der Humboldt-Universität und der außeruniversitären Forschungsinstitute muß so errichtet werden, daß die Hochgeschwindigkeitskommunikation, ausgehend von den Arbeitsplätzen der Wissenschaftler und Studenten, innerhalb der Institute, zwischen verschiedenen Standorten der Universität in Berlin, zu künftigen Partnern des Wissenschafts- und Wirtschaftsstandortes Adlershof sowie im deutschen und in internationalen Wissenschaftsnetzen gesichert ist. Moderne computergestützte Dienste, so zum Beispiel grafik- und multimediaorientierte Informations- und Kommunikationsdienste, implizieren ein dynamisches Wachstum des Volumens und der Geschwindigkeit des Datenverkehrs zwischen den genannten Bereichen. Daher müssen die Infrastruktur der passiven Verkabelung als auch die aktiven Kommunikationsgeräte entsprechend ausgelegt sein.

12. Die moderne Bibliothek sollte nicht nur Service-Einrichtung für die Informationsbeschaffung, -speicherung und -lieferung sein. Sie sollte neben den üblichen Buchfreihandbeständen, Archiven, (computerisierten) Katalogen und Lesesälen auch über Räume mit Arbeitsplätzen mit Terminal- und Druckerinstrumentierung, über Begegnungs- und Kommunikationsräume, Cafeteria und Zeitungsleseplätze verfügen. Gerade die persönliche Begegnung von Wissenschaftlern verschiedener Institute mit Ingenieuren von Wirtschaftsunternehmen wird für einen Hochtechnologie-Standort wie den in Adlershof geplanten wesentlich sein.

13. Anbetracht der gegenwärtigen schnellen Wandlung des gesamten Informationsmanagements können Planungen für ein Informations- und Kommunikationszentrum nicht ohne laufende Präzisierungen durchgeführt werden. Es bedarf der intensiven Beobachtung der weiteren Entwicklung und flexibler innenarchitektonischer Lösungen, um den Anforderungen an der Wende zum nächsten Jahrtausend gerecht zu werden. Gleichzeitig muß das Bibliothekspersonal auf die neuen Anforderungen vorbereitet werden. Der Bibliothekarberuf wird sich erheblich wandeln und ein breites Spektrum an Informatikkenntnissen erfordern.

\section{Anschrift der Autoren:}

Dr. Milan Bulaty

Prof. Dr. Michael Müller-Preußker

Dr. Peter Schirmbacher

Humboldt-Universität

Unter den Linden 6

D-10099 Berlin 